\title{
Uncovering the Surface and Phase Effect of Molybdenum Carbides on Hydrogen Evolution: a First-Principles Study
}

Guang-Qiang Yu ${ }^{1}$, Bo-Ying Huang ${ }^{1}$, Xiaobo Chen $^{1}$, Da Wang ${ }^{2}$, Feipeng Zheng ${ }^{1 *}$, Xi$\mathrm{Bo} \mathrm{Li} i^{1 *}$

1 Siyuan Laboratory, Guangzhou Key Laboratory of Vacuum Coating Technologies and New Energy Materials, Department of Physics, Jinan University, Guangzhou 510632, Guangdong, PR China

2 School of Materials Science and Engineering, Shanghai University, Shanghai 200444, PR China

\section{vdW Effect and Ideal Binding Site for $\mathrm{H}$}

In order to verify the reliability of our DFT results for not considering van der Walls (vdW) interactions in this work, the differential free energy changes of adsorbed $\mathrm{H}$ on different $\mathrm{MoC}$ surfaces while considering the $\mathrm{vdW}\left(\Delta \mathrm{G}_{\mathrm{H}-\mathrm{vdw}}\right)$ or not $\left(\Delta \mathrm{G}_{\mathrm{H}-\mathrm{novdw}}\right.$, or $\Delta \mathrm{G}_{\mathrm{H}}$ in main text) are explored. In the calculated results, the $\Delta \mathrm{G}_{\mathrm{H}-\mathrm{vdw}}$ and $\Delta \mathrm{G}_{\mathrm{H}}$ on top site of $\mathrm{C}$ in $\alpha$-MoC-(100)-C, and on fcc site of Mo in $\alpha-\mathrm{MoC}-(001)-\mathrm{Mo}$ are $-0.67 \mathrm{eV}$ and $-0.67 \mathrm{eV}$, and $-0.64 \mathrm{eV}$ and $-0.61 \mathrm{eV}$, respectively. The difference between $\Delta \mathrm{G}_{\mathrm{H}-\mathrm{vdw}}$ and $\Delta \mathrm{G}_{\mathrm{H}}$ on the corresponding surfaces, $\Delta \mathrm{G}_{\mathrm{H}-\mathrm{vdw}}-\Delta \mathrm{G}_{\mathrm{H}}$, are 0.00 and $0.03 \mathrm{eV}$, respectively. And more, we also explore the vdW effect on different $\mathrm{H}$ coverage. The $\Delta \mathrm{G}_{\mathrm{H}-}$ vdw on top of $\mathrm{C}$ in $\delta$-MoC-(001) is about $0.03,0.02,0.00$ and $0.00 \mathrm{eV}$ higher than that of the corresponding $\Delta \mathrm{G}_{\mathrm{H}}$ at $1 / 8,2 / 8,3 / 8$ and $4 / 8 \mathrm{H}$ ML, respectively. Those results clearly show the hydrogen adsorption ability will be not change too much $(-0.03 \sim 0.03 \mathrm{eV})$ while considering the $\mathrm{vdW}$ or not, indicating the accuracy of our calculations without vdW corrections in this work. In additional, the optB86b-vdW method ${ }^{1}$ is applied for elevating the vdW correction in this part.

The HER activity is strongly related with the adsorption site on one surface, and the ideal binding site for $\mathrm{H}$, which owns $\left|\Delta \mathrm{G}_{\mathrm{H}}\right|<0.20 \mathrm{eV}$, plays a vital role in HER activity. The bare $\delta$ $\mathrm{MoC}(001)$ and bare $\alpha-\mathrm{MoC}-(001)-\mathrm{C}$ are taken as example to explain this. The adsorption order of $\mathrm{H}$ atoms on the two surfaces is as shown in Figure S8 and Figure S9, respectively, and the adsorption ability of $\mathrm{H}\left(\Delta \mathrm{G}_{\mathrm{H}}\right)$ at different $\mathrm{H}$ coverage is shown as Figure 3a. In the $(1 \mathrm{x} 1) \delta$ $\mathrm{MoC}(001)$ surface, there are two possible adsorption site, which could be seen Figure S1, top of $\mathrm{C}_{5}$ and $\mathrm{Mo}_{5}$ site, respectively. The corresponding $\Delta \mathrm{G}_{\mathrm{H}}$ are $-0.22 \mathrm{eV}$ and $0.72 \mathrm{eV}$, respectively, indicting the $\mathrm{H}$ prefer to adsorb on top of $\mathrm{C}_{5}$ site of $\delta-\mathrm{MoC}(001)$. Then the $\Delta \mathrm{G}_{\mathrm{H}}$ at top of $\mathrm{C}_{5}$ site on a $(2 \times 2)$ cell of $\delta-\mathrm{MoC}(001)$ is explored, the corresponding values are $-0.18,-0.17,-0.12$ and $0.17 \mathrm{eV}$, respectively. All their values are in range of $-0.20 \sim 0.20 \mathrm{eV}$, indicating the $\mathrm{C}_{5}$ is the ideal adsorption site for MoC surface. There are only one adsorption site for $\alpha-\mathrm{MoC}-(001)-\mathrm{C}$, top of the $\mathrm{C}_{3}$ site , as shown in Figure 3a. When one $\mathrm{H}$ atom (at $1 \mathrm{H}$ ML) adsorbs on the model of $\alpha-\mathrm{MoC}-(001)-\mathrm{C}$, the $\Delta \mathrm{G}_{\mathrm{H}}$ is $-0.76 \mathrm{eV}$, indicating it poor HER activity. However, the second $\mathrm{H}$ adsorption (at $2 \mathrm{H} \mathrm{ML}$, as shown in Figure S9a) will be much suitable for HER, as the $\Delta \mathrm{G}_{\mathrm{H}}$ becomes $-0.15 \mathrm{eV}$, much close to $0 \mathrm{eV}$. But the further $\mathrm{H}$ adsorption (at $3 \mathrm{H} \mathrm{ML}$, as shown in Figure S9b) will be worse, as the structural deformation leading to larger $\Delta \mathrm{G}_{\mathrm{H}}(0.97 \mathrm{eV})$. Thus, $\mathrm{C}_{5}$ and $\mathrm{C}_{3}$ (second $\mathrm{H}$ adsorption) of clean MoC surfaces will be ideal $\mathrm{H}$ binding site for HER. 
Table. S1 Lattice parameters and cohesive energies of different kinds of molybdenum carbide phases. The "Z" indicates number of formulas in the unit cell. The "Cal", "Exp" and "Pre" indicate the parameters obtained by DFT of this work, former experiment and DFT of previous work, respectively. And the bulk phase diagram is also given in Figure S11.

\begin{tabular}{|c|c|c|c|c|c|c|c|c|c|c|}
\hline \multirow[t]{2}{*}{ System } & \multirow[t]{2}{*}{ Space group } & \multirow[t]{2}{*}{$\mathrm{Z}$} & \multicolumn{7}{|c|}{ Lattice parameters } & \multirow{2}{*}{$\begin{array}{c}E_{\mathrm{coh}} \\
(\mathrm{eV})\end{array}$} \\
\hline & & & & $\mathrm{a} / \AA ̊$ & $\mathrm{~b} / \AA \AA$ & $\mathrm{b} / \AA$ & $\gamma /^{\circ}{ }^{\circ}$ & & $\beta /{ }^{\circ}$ & \\
\hline \multirow[t]{2}{*}{$\delta-\mathrm{MoC}$} & $\mathrm{fm}-3 \mathrm{~m}$ & 4 & Cal & 4.367 & 4.367 & 4.367 & 90 & 90 & 90 & 13.89 \\
\hline & & & Exp & 4.278 & 4.278 & $4.278^{2}$ & & & & $14.45^{3}$ \\
\hline \multirow[t]{2}{*}{$\alpha-\mathrm{MoC}$} & p-6m2 & 1 & Cal & 2.912 & 2.912 & 2.827 & 90 & 90 & 120 & 14.51 \\
\hline & & & Exp & 2.898 & 2.898 & $2.809^{4}$ & & & & \\
\hline \multirow[t]{2}{*}{ G-MoC } & $\mathrm{P} 63 / \mathrm{mmc}$ & 2 & $\mathrm{Cal}$ & 2.958 & 2.958 & 5.387 & 90 & 90 & 120 & 14.24 \\
\hline & & & Pre. & $\delta 1-\mathrm{Mo}$ & $\mathrm{N}$ type, & o data ${ }^{5}$ & & & & \\
\hline \multirow[t]{2}{*}{$\eta-\mathrm{MoC}$} & $\mathrm{P} 63 / \mathrm{mmc}$ & 6 & Cal & 3.055 & 3.055 & 15.414 & 90 & 90 & 120 & 13.99 \\
\hline & & & Exp & 3.00 & 3.00 & $14.58^{6}$ & & & & \\
\hline \multirow[t]{2}{*}{ TiP-MoC } & $\mathrm{P} 63 / \mathrm{mmc}$ & 4 & Cal & 3.001 & 3.001 & 10.801 & 90 & 90 & 120 & 14.11 \\
\hline & & & Exp & 2.932 & 2.932 & $10.970^{4}$ & & & & \\
\hline \multirow[t]{2}{*}{$\beta-\mathrm{Mo}_{2} \mathrm{C}$} & Pbcn & 4 & Cal & 4.735 & 6.055 & 5.230 & 90 & 90 & 90 & 21.02 \\
\hline & & & Exp & 4.729 & 6.028 & $5.197^{7}$ & & & & \\
\hline \multirow[t]{3}{*}{$\alpha-\mathrm{Mo}_{2} \mathrm{C}$} & $\mathrm{P} 63 / \mathrm{mmc}$ & 4 & Cal & 6.061 & 6.061 & 4.704 & 90 & 90 & 120 & 21.02 \\
\hline & & & Pre & 6.02 & 6.02 & $4.74^{8}$ & & & & \\
\hline & & & Exp & 3.002 & 3.002 & $4.724^{6}$ & & & & \\
\hline
\end{tabular}

Table S2. The free energies of $\mathrm{H}$ adsorption at different sites on the $\delta-\mathrm{MoC}$ surfaces as shown in Figure S1. The "T", B, "H" and "F" indicate the top, bridge, hep and fcc site, respectively. The "-$/ m$ " indicates $\mathrm{H}$ atom is not stable at the corresponding site, and will move to the " $\mathrm{m}$ " site.

\begin{tabular}{|c|c|c|c|c|c|c|c|c|c|c|}
\hline & \multicolumn{2}{|c|}{ (001) } & \multicolumn{3}{|c|}{ (110) } & \multicolumn{3}{|c|}{ (111)-Mo } & \multicolumn{2}{|c|}{$(111)-C$} \\
\hline Term. & \multicolumn{2}{|c|}{$\mathrm{C}_{5}, \mathrm{Mo}_{5}$} & \multicolumn{3}{|c|}{$\mathrm{C}_{4}, \mathrm{Mo}_{4}$} & \multicolumn{3}{|c|}{$\mathrm{Mo}_{3}$} & \multicolumn{2}{|c|}{$\mathrm{C}_{3}$} \\
\hline No. & 1 & 2 & 1 & 2 & 3 & 1 & 2 & 3 & 1 & $2 / 3$ \\
\hline Site & $\begin{array}{c}\mathrm{T}- \\
\mathrm{Mo}_{5}\end{array}$ & $\mathrm{~T}-\mathrm{C}_{5}$ & $\mathrm{~T}-\mathrm{C}_{4}$ & $\mathrm{~B}-\mathrm{Mo}_{4}$ & $\begin{array}{c}\mathrm{T}- \\
\mathrm{Mo}_{4}\end{array}$ & $\begin{array}{c}\mathrm{T}- \\
\mathrm{Mo}_{3}\end{array}$ & $\mathrm{~F}-\mathrm{Mo}_{3}$ & $\mathrm{H}-\mathrm{Mo}_{3}$ & $\mathrm{~T}-\mathrm{C}_{3}$ & $\mathrm{~F} / \mathrm{H}$ \\
\hline$\Delta G_{\mathrm{H}}(\mathrm{eV})$ & 0.72 & -0.22 & -0.65 & -0.39 & $--/ 2$ & -0.30 & -0.73 & -0.53 & -0.74 & $--/ 1$ \\
\hline Bond. Å & 1.738 & 1.111 & 1.114 & $1.853,2.059$ & & 1.733 & $2.021,2.056,2.059$ & $2.080,2.081,2.082$ & 1.096 & \\
\hline
\end{tabular}

Table S3. The free energies of $\mathrm{H}$ adsorption at different sites on the $\alpha-\mathrm{MoC}$ surfaces as shown in Figure S2. Computed surface phase diagram for (a) (110) and (b) (100)-C of $\alpha-\mathrm{MoC}$ in aqueous solution is shown in Figure S12.

\begin{tabular}{|c|c|c|c|c|c|c|c|c|c|c|}
\hline & \multicolumn{2}{|c|}{$(001)-C$} & \multicolumn{3}{|c|}{ (001)-Mo } & $(100)-C$ & \multicolumn{4}{|c|}{ (110) } \\
\hline Term. & \multicolumn{2}{|c|}{$\mathrm{C}_{3}$} & \multicolumn{3}{|c|}{$\mathrm{Mo}_{3}$} & $\mathrm{C}_{4}$ & \multicolumn{4}{|c|}{$\mathrm{C}_{4}, \mathrm{Mo}_{4}$} \\
\hline No. & 1 & $2 / 3$ & 1 & 2 & 3 & 1 & 1 & 2 & 3 & 4 \\
\hline Site & $\mathrm{T}-\mathrm{C}_{3}$ & $\mathrm{~F} / \mathrm{H}$ & $\mathrm{H}$ & $\mathrm{F}$ & $\mathrm{T}$ & $\mathrm{T}-\mathrm{C}_{4}$ & $\mathrm{~T}-\mathrm{C}_{4}$ & $\begin{array}{c}\mathrm{B}-\mathrm{Mo}_{4}, \\
\mathrm{Mo}_{6}\end{array}$ & $\mathrm{~B}-\mathrm{Mo}_{4}$ & $\mathrm{~T}-\mathrm{Mo}_{4}$ \\
\hline$\Delta G_{\mathrm{H}}(\mathrm{eV})$ & -0.76 & $--/ 1$ & -0.39 & -0.61 & $--/ 2$ & -0.67 & -0.09 & -0.10 & -0.53 & $--/ 3$ \\
\hline Bond. Å & 1.098 & & $\begin{array}{l}1.995, \\
1.995,\end{array}$ & $\begin{array}{l}1.963, \\
1.963,\end{array}$ & & 1.116 & 1.123 & $\begin{array}{l}1.885 \\
1.878\end{array}$ & $\begin{array}{l}1.914 \\
1.914\end{array}$ & \\
\hline
\end{tabular}




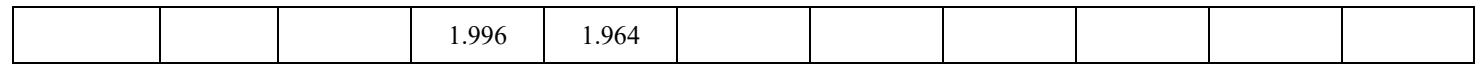

Table S4. The free energies of $\mathrm{H}$ adsorption at different sites on the $\eta-\mathrm{MoC}$ surfaces as shown in Figure S3.

\begin{tabular}{|c|c|c|c|c|c|c|c|}
\hline & \multicolumn{3}{|c|}{ (001)-Mo } & $(001)-\mathrm{C}$ & \multicolumn{3}{|c|}{ (110) } \\
\hline Term. & \multicolumn{3}{|c|}{$\mathrm{Mo}_{3}$} & $\mathrm{C}_{3}$ & \multicolumn{3}{|c|}{$\mathrm{C}_{4}, \mathrm{Mo}_{4}$} \\
\hline No. & 1 & 2 & 3 & 1 & 1 & 2 & 3 \\
\hline Site & $\mathrm{T}$ & $\mathrm{F}$ & $\mathrm{H}$ & $\mathrm{T}$ & $\mathrm{T}-\mathrm{C}_{4}$ & $\mathrm{~B}-\mathrm{Mo}_{4}$ & $\mathrm{~B}-\mathrm{Mo}_{4}$ \\
\hline$\Delta G_{\mathrm{H}}(\mathrm{eV})$ & -0.15 & -0.65 & -0.48 & -0.82 & -0.35 & -0.32 & -0.15 \\
\hline \multirow[t]{2}{*}{ Bond. Å } & 1.743 & $\begin{array}{c}2.036, \\
2.040,2.040\end{array}$ & $\begin{array}{c}-2.080, \\
2.080,- \\
2.081\end{array}$ & 1.157 & 1.117 & $\begin{array}{l}1.907 \\
1.977\end{array}$ & $\begin{array}{l}1.944 \\
1.946\end{array}$ \\
\hline & \multicolumn{7}{|c|}{$(0-14)$} \\
\hline Term. & \multicolumn{7}{|c|}{$\mathrm{C}_{3}, \mathrm{C}_{4}, \mathrm{C}_{5}, \mathrm{Mo}_{4}, \mathrm{Mo}_{5}$} \\
\hline No. & 1 & 2 & 3 & 4 & 5 & 6 & 7 \\
\hline Site & $\mathrm{T}-\mathrm{C}_{3}$ & $\mathrm{~T}-\mathrm{C}_{4}$ & $\mathrm{~T}-\mathrm{C}_{5}$ & $T-C_{5}$ & $T-C_{5}$ & $\begin{array}{c}\mathrm{B}-\mathrm{Mo}_{4}, \\
\mathrm{Mo}_{5}\end{array}$ & $\mathrm{H}-\mathrm{Mo}_{4}$ \\
\hline$\Delta G_{\mathrm{H}}(\mathrm{eV})$ & -0.84 & -0.49 & 0.14 & -0.05 & 0.23 & -0.48 & -0.27 \\
\hline Bond. Å & 1.096 & 1.114 & 1.124 & 1.113 & 1.122 & $1.930,2.090$ & $2.069,2.103,2.181$ \\
\hline
\end{tabular}

Table S5. The free energies of $\mathrm{H}$ adsorption on different sites of the G-MoC surfaces as shown in Figure S4.

\begin{tabular}{|c|c|c|c|c|c|c|c|c|}
\hline & \multicolumn{3}{|c|}{ (010) } & \multicolumn{2}{|c|}{ (110) } & \multicolumn{2}{|c|}{ (001)-Mo } & $(001)-$ \\
\hline Term. & \multicolumn{3}{|c|}{$\mathrm{C}_{5}, \mathrm{Mo}_{4}$} & \multicolumn{2}{|c|}{$\mathrm{C}_{4}, \mathrm{Mo}_{4}$} & \multicolumn{2}{|c|}{$\mathrm{Mo}_{3}$} & $\mathrm{C}_{3}$ \\
\hline No. & 1 & 2 & 3 & 1 & 2 & 1 & 2 & 1 \\
\hline Site & $\mathrm{T}-\mathrm{Mo}_{4}$ & $T-C_{5}$ & $\mathrm{~B}-\mathrm{Mo}_{4}$ & $\mathrm{~T}-\mathrm{C}_{4}$ & $\mathrm{~B}-\mathrm{Mo}_{4}$ & $\mathrm{H}$ & $\mathrm{F}$ & $\mathrm{T}$ \\
\hline$\Delta G_{\mathrm{H}}(\mathrm{eV})$ & 0.04 & 0.48 & $--/ 1$ & -0.26 & -0.66 & -0.33 & -0.49 & -0.95 \\
\hline Bond. Å & 1.727 & 1.169 & & 1.116 & $1.953,1.955$ & $2.035,2.035,2.037$ & $1.976,1.976,1.976$ & 1.095 \\
\hline
\end{tabular}

Table S6. The adsorption free energies of $\mathrm{H}$ at different sites on the TiP-MoC surfaces as shown in Figure S5.

\begin{tabular}{|c|c|c|c|c|c|c|c|c|c|}
\hline & \multicolumn{4}{|c|}{ (010) } & \multicolumn{4}{|c|}{ (110) } & \\
\hline Term. & \multicolumn{4}{|c|}{$\mathrm{C}_{4}, \mathrm{C}_{5}, \mathrm{Mo}_{5}$} & \multicolumn{4}{|c|}{$\mathrm{C}_{4}, \mathrm{Mo}_{4}$} & \\
\hline No. & 1 & 2 & 3 & 4 & 1 & 2 & 3 & 4 & \\
\hline Site & $\mathrm{T}-\mathrm{C}_{4}$ & $\mathrm{~T}-\mathrm{C}_{5}$ & $\mathrm{~B}-\mathrm{Mo}_{5}$ & $\mathrm{~T}-\mathrm{Mo}_{5}$ & $T-C_{4}$ & $\mathrm{~T}-\mathrm{C}_{4}$ & $\mathrm{~B}-\mathrm{Mo}_{4}$ & $\mathrm{~B}-\mathrm{Mo}_{4}$ & \\
\hline$\Delta G_{\mathrm{H}}(\mathrm{eV})$ & -0.71 & -0.08 & 0.33 & 0.46 & -1.23 & -0.36 & -0.31 & -0.06 & \\
\hline Bond. Å & 1.109 & 1.113 & $1.938,1.939$ & 1.724 & 1.103 & 1.127 & $1.925,1.928$ & $1.931,1.939$ & \\
\hline Sur. & \multicolumn{9}{|c|}{ (014) } \\
\hline Term. & \multicolumn{9}{|c|}{$\mathrm{C}_{4}, \mathrm{C}_{5}, \mathrm{Mo}_{4}, \mathrm{Mo}_{5}$} \\
\hline No. & 1 & 2 & 3 & 4 & 5 & 6 & 7 & 8 & 9 \\
\hline Site & $T-C_{4}$ & $\mathrm{~T}-\mathrm{C}_{5}$ & $\mathrm{~T}-\mathrm{C}_{5}$ & $T-C_{4}$ & $\mathrm{~B}-\mathrm{Mo}_{4}$ & $\mathrm{~B}-\mathrm{Mo}_{4}$ & $\mathrm{~T}-\mathrm{Mo}_{4}$ & $\mathrm{~T}-\mathrm{Mo}_{5}$ & $\mathrm{~T}-\mathrm{Mo}_{5}$ \\
\hline$\Delta G_{\mathrm{H}}(\mathrm{eV})$ & -0.55 & 1.37 & 0.06 & -0.60 & -0.17 & -0.50 & 0.15 & 0.67 & 0.54 \\
\hline Bond. Å & 1.103 & 1.193 & 1.117 & 1.139 & $1.950,1.950$ & $1.935,1.936$ & 1.736 & 1.742 & 1.729 \\
\hline
\end{tabular}


Table S7. The adsorption free energies of $\mathrm{H}$ at different sites on the $\beta-\mathrm{Mo}_{2} \mathrm{C}$ surfaces as shown in Figure S6.

\begin{tabular}{|c|c|c|c|c|c|c|c|c|}
\hline Sur. & \multicolumn{3}{|c|}{ (100)-Mo } & \multicolumn{3}{|c|}{$(100)-C$} & \multicolumn{2}{|c|}{$(001)-C$} \\
\hline Term. & \multicolumn{3}{|c|}{$\mathrm{Mo}_{1}, \mathrm{Mo}_{2}$} & \multicolumn{3}{|c|}{$\mathrm{C}_{3}, \mathrm{Mo}_{3}$} & \multicolumn{2}{|c|}{$\mathrm{C}_{3}, \mathrm{Mo}_{3}$} \\
\hline No. & 1 & 2 & 3 & 1 & 2 & 3 & 1 & 2 \\
\hline Site & $\mathrm{H}-1 \mathrm{Mo}_{2} 2 \mathrm{Mo}_{1}$ & $\mathrm{H}-2 \mathrm{Mo}_{2} 1 \mathrm{Mo}_{1}$ & $\mathrm{H}-1 \mathrm{Mo}_{2} 2 \mathrm{Mo}_{1}$ & $T-C_{3}$ & $\mathrm{H}-\mathrm{Mo}_{3}$ & $\mathrm{~B}-\mathrm{Mo}_{3}$ & $\begin{array}{l}\mathrm{T}- \\
\mathrm{C}_{3}\end{array}$ & $\mathrm{~B}-\mathrm{Mo}_{3}$ \\
\hline$\Delta G_{\mathrm{H}}(\mathrm{eV})$ & -0.83 & -0.57 & -0.75 & -0.45 & 0.08 & 0.02 & -0.86 & -0.07 \\
\hline Bond. Å & $\begin{array}{c}\text { 1.958, } 2.007 \\
2.014\end{array}$ & $\begin{array}{c}1.978,2.046 \\
2.054\end{array}$ & $\begin{array}{c}2.003,2.060 \\
2.027\end{array}$ & 1.100 & $\begin{array}{c}1.824,2.115 \\
2.164\end{array}$ & $\begin{array}{l}1.912 \\
1.972\end{array}$ & 1.103 & $\begin{array}{l}1.838 \\
1.894\end{array}$ \\
\hline Sur. & \multicolumn{3}{|c|}{$(111)-\mathrm{C}$} & (110) -Mo & \multicolumn{4}{|c|}{$110-\mathrm{C}$} \\
\hline Term. & \multicolumn{3}{|c|}{$\mathrm{C}_{3}, \mathrm{C}_{4}, \mathrm{Mo}_{3}$} & $\mathrm{Mo}_{3}$ & \multicolumn{4}{|c|}{$\mathrm{C}_{4}, \mathrm{C}_{5}, \mathrm{Mo}_{3}$} \\
\hline No. & 1 & 2 & 3 & 1 & 1 & 2 & 3 & 4 \\
\hline Site & $\mathrm{T}-\mathrm{C}_{3}$ & $\mathrm{~T}-\mathrm{C}_{4}$ & $\mathrm{~B}-\mathrm{Mo}_{3}$ & $\mathrm{~F}$ & $T-C_{4}$ & $T-C_{5}$ & $\begin{array}{c}\mathrm{T}- \\
\mathrm{Mo}_{3}\end{array}$ & $\mathrm{~B}-\mathrm{Mo}_{3}$ \\
\hline$\Delta G_{\mathrm{H}}(\mathrm{eV})$ & -0.51 & -0.23 & 0.35 & -0.60 & -0.11 & 0.63 & 0.28 & 0.16 \\
\hline Bond. Å & 1.104 & 1.113 & $1.876,1.929$ & $\begin{array}{c}1.927,2.218 \\
2.266\end{array}$ & 1.120 & 1.175 & 1.726 & $\begin{array}{l}1.926 \\
1.953\end{array}$ \\
\hline
\end{tabular}

Table S8. The adsorption free energies of $\mathrm{H}$ at different sites on the $\alpha-\mathrm{Mo}_{2} \mathrm{C}$ surfaces as shown in Figure S7. The H on FCC and HCP site of (001)-C and (100)-C is not stable, and will move to top site after relaxion.

\begin{tabular}{|c|c|c|c|c|c|c|c|c|}
\hline & $(001)-C$ & $(100)-C$ & \multicolumn{3}{|c|}{ (001)-Mo } & \multicolumn{3}{|c|}{$(011)-\mathrm{C}$} \\
\hline Term. & $\mathrm{C}_{3}$ & $\mathrm{C}_{3}$ & \multicolumn{3}{|c|}{$\mathrm{Mo}_{1}, \mathrm{Mo}_{2}$} & \multicolumn{3}{|c|}{$\mathrm{C}_{4}, \mathrm{C}_{5}, \mathrm{Mo}_{3}$} \\
\hline No. & 1 & 1 & 1 & 2 & 3 & 1 & 2 & 3 \\
\hline Site & $\mathrm{T}-\mathrm{C}_{3}$ & $\mathrm{~T}-\mathrm{C}_{3}$ & $\mathrm{H}-2 \mathrm{Mo}_{2} 1 \mathrm{Mo}_{1}$ & $\mathrm{H}-1 \mathrm{Mo}_{2} 2 \mathrm{Mo}_{1}$ & $\mathrm{~F}-2 \mathrm{Mo}_{1} 1 \mathrm{Mo}_{2}$ & $\mathrm{~T}-\mathrm{C}_{4}$ & $\mathrm{~T}-\mathrm{C}_{5}$ & $\mathrm{~T}-\mathrm{Mo}_{3}$ \\
\hline$\Delta G_{\mathrm{H}}(\mathrm{eV})$ & -0.34 & -0.72 & -0.83 & -0.80 & -0.85 & -0.30 & 0.78 & $--/ 1$ \\
\hline Bond. Å & 1.103 & 1.102 & $2.018,2.042,2.043$ & $2.021,2.022,2.055$ & $1.954,2.015,2.015$ & 1.125 & 1.147 & \\
\hline
\end{tabular}

Table S9. Free energy change of $* \mathrm{O}$ or $* \mathrm{OH}$ at adsorption site on $\delta-\mathrm{MoC}$ surfaces as shown in Figure S1. The white balls indicate the adsorption site for $\mathrm{O}$ and $\mathrm{OH}$. The number $m$ is the same as $\mathrm{H}$ adsorption above, and the grey parts indicate that the corresponding surface is free of *O and $* \mathrm{OH}$.

\begin{tabular}{|c|c|c|c|c|c|c|c|c|c|c|}
\hline & \multicolumn{2}{|c|}{$(001)$} & \multicolumn{3}{c|}{$(110)$} & \multicolumn{3}{c|}{$(111)-\mathrm{Mo}$} & \multicolumn{2}{c|}{$\mathrm{MO}_{3}$} \\
\hline Term. & \multicolumn{2}{|c|}{$\mathrm{C}_{5}, \mathrm{Mo}_{5}$} & \multicolumn{3}{|c|}{$\mathrm{C}_{4}, \mathrm{Mo}_{4}$} & \multicolumn{3}{c|}{$\mathrm{C}_{3}$} \\
\hline No. & 1 & 2 & 1 & 2 & 3 & 1 & 2 & 3 & 1 & $2 / 3$ \\
\hline Site & $\mathrm{T}-$ & $\mathrm{T}-\mathrm{C}_{5}$ & $\mathrm{~T}-\mathrm{C}_{4}$ & $\mathrm{~B}-\mathrm{Mo}_{4}$ & $\mathrm{~T}-$ & $\mathrm{T}-$ & $\mathrm{F}-\mathrm{Mo}_{3}$ & $\mathrm{H}-\mathrm{Mo}_{3}$ & $\mathrm{~T}-\mathrm{C}_{3}$ & $\mathrm{~F} / \mathrm{H}$ \\
& $\mathrm{Mo}_{5}$ & & & & $\mathrm{Mo}_{4}$ & $\mathrm{Mo}_{3}$ & & & & \\
\hline$\Delta G_{\mathrm{O}}(\mathrm{eV})$ & 0.21 & 0.51 & -0.77 & -0.12 & $--/ 2$ & -0.26 & -1.40 & -1.39 & 1.36 & $-0.80 /-0.88$ \\
\hline$\Delta G_{\mathrm{OH}}(\mathrm{eV})$ & 0.74 & 0.59 & -0.08 & -0.68 & & -0.44 & -1.19 & -1.19 & 0.29 & $-0.04 /-0.06$ \\
\hline
\end{tabular}


Table S10. Free energy changes of ${ }^{*} \mathrm{O}$ or ${ }^{*} \mathrm{OH}$ at adsorption site on $\alpha-\mathrm{MoC}$ surfaces as shown in Figure S2.

\begin{tabular}{|c|c|c|c|c|c|c|c|c|c|c|}
\hline & \multicolumn{2}{|c|}{$(001)-\mathrm{C}$} & \multicolumn{3}{|c|}{ (001)-Mo } & $(100)-C$ & \multicolumn{4}{|c|}{ (110) } \\
\hline Term. & \multicolumn{2}{|c|}{$\mathrm{C}_{3}$} & \multicolumn{3}{|c|}{$\mathrm{Mo}_{3}$} & $\mathrm{C}_{4}$ & \multicolumn{4}{|c|}{$\mathrm{C}_{4}, \mathrm{Mo}_{4}$} \\
\hline No. & 1 & $2 / 3$ & 1 & 2 & 3 & 1 & 1 & 2 & 3 & 4 \\
\hline Site & $\mathrm{T}-\mathrm{C}_{3}$ & $\mathrm{~F}$ & $\mathrm{H}$ & F & $\mathrm{T}$ & $\mathrm{T}-\mathrm{C}_{4}$ & $\mathrm{~T}-\mathrm{C}_{4}$ & $\begin{array}{c}\mathrm{B}-\mathrm{Mo}_{4}, \\
\mathrm{Mo}_{6}\end{array}$ & $\mathrm{~B}-\mathrm{Mo}_{4}$ & $\mathrm{~T}-\mathrm{Mo}_{4}$ \\
\hline$\Delta G_{\mathrm{O}}(\mathrm{eV})$ & - & 3.32 & -1.47 & -0.97 & $--/ 2$ & 0.37 & & & -0.41 & \\
\hline$\Delta G_{\mathrm{OH}}(\mathrm{eV})$ & - & 3.88 & -0.8 & -0.43 & & 0.36 & & & -0.68 & \\
\hline
\end{tabular}

Table S11. Free energy changes of $* \mathrm{O}$ or $* \mathrm{OH}$ at adsorption site on $\mathrm{G}-\mathrm{MoC}$ surfaces as shown in Figure S3.

\begin{tabular}{|c|c|c|c|c|c|c|c|c|c|c|}
\hline & \multicolumn{3}{|c|}{$(010)$} & \multicolumn{2}{c|}{$(110)$} & \multicolumn{2}{c|}{$(001)-\mathrm{Mo}$} & \multicolumn{3}{c|}{$(001)-\mathrm{C}$} \\
\hline Term. & \multicolumn{3}{|c|}{$\mathrm{C}_{5}, \mathrm{Mo}_{4}$} & \multicolumn{2}{c|}{$\mathrm{C}_{4}, \mathrm{Mo}_{4}$} & \multicolumn{2}{|c|}{$\mathrm{Mo}_{3}$} & \multicolumn{2}{c|}{$\mathrm{C}_{3}$} & \\
\hline No. & 1 & 2 & 3 & 1 & 2 & 1 & 2 & 1 & $\mathrm{H}$ \\
\hline Site & $\mathrm{T}-$ & $T-C_{5}$ & $\mathrm{~B}-$ & $\mathrm{T}-\mathrm{C}_{4}$ & $\mathrm{~B}-\mathrm{Mo}_{4}$ & $\mathrm{H}$ & $\mathrm{F}$ & $\mathrm{T}$ & $\mathrm{F}$ & \\
& $\mathrm{Mo}_{4}$ & & $\mathrm{Mo}_{4}$ & & & & & & -0.71 & -0.57 \\
\hline$\Delta G_{\mathrm{O}}(\mathrm{eV})$ & -0.34 & & & 0.11 & -0.85 & -0.58 & -0.58 & -0.60 & -0.36 & -0.35 \\
\hline$\Delta G_{\mathrm{OH}}(\mathrm{eV})$ & -0.84 & & & 0.58 & -0.79 & -0.45 & -0.45 & -0.34 & \\
\hline
\end{tabular}

Table S12. Free energy changes of $* \mathrm{O}$ or $* \mathrm{OH}$ at adsorption site on $\eta-\mathrm{MoC}$ surfaces as shown in Figure S4.

\begin{tabular}{|c|c|c|c|c|c|c|c|}
\hline & \multicolumn{3}{|c|}{ (001)-Mo } & $(001)-\mathrm{C}$ & \multicolumn{3}{|c|}{ (110) } \\
\hline Term. & \multicolumn{3}{|c|}{$\mathrm{Mo}_{3}$} & $\mathrm{C}_{3}$ & \multicolumn{3}{|c|}{$\mathrm{C}_{4}, \mathrm{Mo}_{4}$} \\
\hline No. & 1 & 2 & 3 & 1 & 1 & 2 & 3 \\
\hline Site & $\mathrm{T}$ & $\mathrm{F}$ & $\mathrm{H}$ & $\mathrm{T}$ & $\mathrm{T}-\mathrm{C}_{4}$ & $\mathrm{~B}-\mathrm{Mo}_{4}$ & $\mathrm{~B}-\mathrm{Mo}_{4}$ \\
\hline$\Delta G_{\mathrm{O}}(\mathrm{eV})$ & -1.17 & -1.14 & -1.17 & -0.67 & -0.34 & 0.25 & -0.37 \\
\hline \multirow[t]{2}{*}{$\Delta G_{\mathrm{OH}}(\mathrm{eV})$} & -0.22 & -1.01 & -0.38 & -0.21 & 0.27 & -0.37 & -0.25 \\
\hline & \multicolumn{7}{|c|}{$(0-14)$} \\
\hline Term. & \multicolumn{7}{|c|}{$\mathrm{C}_{3}, \mathrm{C}_{4}, \mathrm{C}_{5}, \mathrm{Mo}_{4}, \mathrm{Mo}_{5}$} \\
\hline No. & 1 & 2 & 3 & 4 & 5 & 6 & 7 \\
\hline Site & $\mathrm{T}-\mathrm{C}_{3}$ & $\mathrm{~T}-\mathrm{C}_{4}$ & $\mathrm{~T}-\mathrm{C}_{5}$ & $T-C_{5}$ & $T-C_{5}$ & $\begin{array}{c}\mathrm{B}-\mathrm{Mo}_{4} \\
\mathrm{Mo}_{5}\end{array}$ & $\mathrm{H}-\mathrm{Mo}_{4}$ \\
\hline$\Delta G_{\mathrm{O}}(\mathrm{eV})$ & -1.62 & & & & & -1.12 & -0.54 \\
\hline$\Delta G_{\mathrm{OH}}(\mathrm{eV})$ & -0.24 & & & & & -0.67 & -0.40 \\
\hline
\end{tabular}

Table S13. Free energy changes of $* \mathrm{O}$ or $* \mathrm{OH}$ at adsorption site on TiP-MoC surfaces as shown in Figure S5.

\begin{tabular}{|l|l|l|l|}
\hline & $(010)$ & $(110)$ & \\
\hline
\end{tabular}




\begin{tabular}{|c|c|c|c|c|c|c|c|c|c|}
\hline Term. & \multicolumn{7}{|c|}{$\mathrm{C}_{4}, \mathrm{C}_{5}, \mathrm{Mo}_{5}$} & \multicolumn{5}{c|}{$\mathrm{C}_{4}, \mathrm{Mo}_{4}$} & \\
\hline No. & 1 & 2 & 3 & 4 & 1 & 2 & 3 & 4 & \\
\hline Site & $\mathrm{T}-\mathrm{C}_{4}$ & $\mathrm{~T}-\mathrm{C}_{5}$ & $\mathrm{~B}-\mathrm{Mo}_{5}$ & $\mathrm{~T}-\mathrm{Mo}_{5}$ & $T-C_{4}$ & $\mathrm{~T}-\mathrm{C}_{4}$ & $\mathrm{~B}-\mathrm{Mo}_{4}$ & $\mathrm{~B}-\mathrm{Mo}_{4}$ & \\
\hline$\Delta G_{\mathrm{O}}(\mathrm{eV})$ & -0.32 & 0.57 & 0.56 & & -0.03 & -0.68 & -0.38 & -0.03 & \\
\hline$\Delta G_{\mathrm{OH}}(\mathrm{eV})$ & 0.19 & 0.95 & 0.38 & & -0.37 & 0.41 & -0.40 & -0.37 & \\
\hline Sur. & \multicolumn{7}{|c|}{$(014)$} \\
\hline Term. & \multicolumn{7}{|c|}{$\mathrm{C}_{4}, \mathrm{C}_{5}, \mathrm{Mo}_{4}, \mathrm{Mo}_{5}$} & \multicolumn{1}{c|}{} \\
\hline No. & 1 & 2 & 3 & 4 & 5 & 6 & 7 & 8 & 9 \\
\hline Site & $T-C_{4}$ & $\mathrm{~T}-\mathrm{C}_{5}$ & $\mathrm{~T}-\mathrm{C}_{5}$ & $T-C_{4}$ & $\mathrm{~B}-\mathrm{Mo}_{4}$ & $\mathrm{~B}-\mathrm{Mo}_{4}$ & $\mathrm{~T}-\mathrm{Mo}_{4}$ & $\mathrm{~T}-\mathrm{Mo}_{5}$ & $\mathrm{~T}-\mathrm{Mo}_{5}$ \\
\hline$\Delta G_{\mathrm{O}}(\mathrm{eV})$ & -0.45 & & 0.86 & -1.20 & -0.24 & -0.84 & & & \\
\hline$\Delta G_{\mathrm{OH}}(\mathrm{eV})$ & 0.14 & & 1.12 & 0.01 & -0.52 & -1.06 & & & \\
\hline
\end{tabular}

Table S14. Free energy changes of * $\mathrm{O}$ or $* \mathrm{OH}$ at adsorption site on $\beta-\mathrm{Mo}_{2} \mathrm{C}$ surfaces as shown in Figure S6.

\begin{tabular}{|c|c|c|c|c|c|c|c|c|}
\hline Sur. & \multicolumn{3}{|c|}{ (100)-Mo } & \multicolumn{3}{|c|}{$(100)-C$} & \multicolumn{2}{|c|}{$(001)-C$} \\
\hline Term. & \multicolumn{3}{|c|}{$\mathrm{Mo}_{1}, \mathrm{Mo}_{2}$} & \multicolumn{3}{|c|}{$\mathrm{C}_{3}, \mathrm{Mo}_{3}$} & \multicolumn{2}{|c|}{$\mathrm{C}_{3}, \mathrm{Mo}_{3}$} \\
\hline No. & 1 & 2 & 3 & 1 & 2 & 3 & 1 & 2 \\
\hline Site & $\mathrm{H}-1 \mathrm{Mo}_{2} 2 \mathrm{Mo}_{1}$ & $\mathrm{H}-2 \mathrm{Mo}_{2} 1 \mathrm{Mo}_{1}$ & $\mathrm{H}-1 \mathrm{Mo}_{2} 2 \mathrm{Mo}_{1}$ & $T-C_{3}$ & $\mathrm{H}-\mathrm{Mo}_{3}$ & $\mathrm{~B}-\mathrm{Mo}_{3}$ & $\begin{array}{c}\mathrm{T}- \\
\mathrm{C}_{3}\end{array}$ & $\mathrm{~B}-\mathrm{Mo}_{3}$ \\
\hline$\Delta G_{0}(\mathrm{eV})$ & & & & -0.67 & & 0.16 & 0.02 & 0.70 \\
\hline$\Delta G_{\mathrm{OH}}(\mathrm{eV})$ & & & & 0.13 & & -0.11 & 1.43 & 0.54 \\
\hline Sur. & \multicolumn{3}{|c|}{ (111)-C } & (110) -Mo & \multicolumn{4}{|c|}{$110-\mathrm{C}$} \\
\hline Term. & \multicolumn{3}{|c|}{$\mathrm{C}_{3}, \mathrm{C}_{4}, \mathrm{Mo}_{3}$} & $\mathrm{Mo}_{3}$ & \multicolumn{4}{|c|}{$\mathrm{C}_{4}, \mathrm{C}_{5}, \mathrm{Mo}_{3}$} \\
\hline No. & 1 & 2 & 3 & 1 & 1 & 2 & 3 & 4 \\
\hline Site & $\mathrm{T}-\mathrm{C}_{3}$ & $\mathrm{~T}-\mathrm{C}_{4}$ & $\mathrm{~B}-\mathrm{Mo}_{3}$ & $\mathrm{~F}$ & $T-C_{4}$ & $T-C_{5}$ & $\begin{array}{c}\mathrm{T}- \\
\mathrm{Mo}_{3}\end{array}$ & $\mathrm{~B}-\mathrm{Mo}_{3}$ \\
\hline$\Delta G_{\mathrm{O}}(\mathrm{eV})$ & 0.48 & 0.60 & 0.48 & -1.65 & -0.07 & & & 0.32 \\
\hline$\Delta G_{\mathrm{OH}}(\mathrm{eV})$ & 0.44 & 0.48 & -0.27 & -1.04 & 0.72 & & & -0.07 \\
\hline
\end{tabular}

Table S15. Free energy changes of $* \mathrm{O}$ or $* \mathrm{OH}$ at adsorption site on $\alpha-\mathrm{Mo}_{2} \mathrm{C}$ surfaces as shown in Figure S7.

\begin{tabular}{|c|c|c|c|c|c|c|c|c|}
\hline & $(001)-C$ & $(100)-\mathrm{C}$ & \multicolumn{3}{|c|}{ (001)-Mo } & \multicolumn{3}{|c|}{ (011)-C } \\
\hline Term. & $\mathrm{C}_{3}$ & $\mathrm{C}_{3}$ & \multicolumn{3}{|c|}{$\mathrm{Mo}_{1}, \mathrm{Mo}_{2}$} & \multicolumn{3}{|c|}{$\mathrm{C}_{4}, \mathrm{C}_{5}, \mathrm{Mo}_{3}$} \\
\hline No. & 1 & 1 & 1 & 2 & 3 & 1 & 2 & 3 \\
\hline Site & $\mathrm{T}-\mathrm{C}_{3}$ & $\mathrm{~T}-\mathrm{C}_{3} / \mathrm{b}$ & $\mathrm{H}-2 \mathrm{Mo}_{2} 1 \mathrm{Mo}_{1}$ & $\mathrm{H}-1 \mathrm{Mo}_{2} 2 \mathrm{Mo}_{1}$ & $\mathrm{~F}-2 \mathrm{Mo}_{1} 1 \mathrm{Mo}_{2}$ & $\mathrm{~T}-\mathrm{C}_{4}$ & $\mathrm{~T}-\mathrm{C}_{5}$ & B- $\mathrm{C}_{4}$ \\
\hline$\Delta G_{\mathrm{O}}(\mathrm{eV})$ & -0.26 & $\begin{array}{c}-0.03 /- \\
0.03\end{array}$ & -1.79 & -1.54 & -2.02 & 0.73 & 0.73 & -0.11 \\
\hline$\Delta G_{\mathrm{OH}}(\mathrm{eV})$ & 0.14 & $0.18 / 0.17$ & -1.29 & -1.05 & -1.07 & 1.09 & 0.70 & 0.39 \\
\hline
\end{tabular}

Table S16. Free energy changes of ${ }^{*} \mathrm{O}$ or ${ }^{*} \mathrm{OH}$ at adsorption site on MoC surfaces at different coverages. The unit is in $\mathrm{eV}$, and the "...." the considered coverage does not exist on the slab model in our calculations.

\begin{tabular}{|l|l|l|l|l|}
\hline $\mathrm{ML}$ & $1 / 4$ & $1 / 2$ & $3 / 4$ & 1 \\
\hline
\end{tabular}




\begin{tabular}{|c|c|c|c|c|}
\hline$\delta-\mathrm{MoC}-(111)-\mathrm{Mo}$ & $-1.40\left({ }^{*} \mathrm{O}\right)$ & $-0.76\left({ }^{*} \mathrm{O}\right)$ & $-0.72\left({ }^{*} \mathrm{OH}\right)$ & $-0.08\left({ }^{*} \mathrm{OH}\right)$ \\
\hline$\delta-\mathrm{MoC}-(110)$ & --- & $-0.68\left({ }^{*} \mathrm{OH}\right)$ & -- & $-0.20\left({ }^{*} \mathrm{OH}\right)$ \\
\hline$\eta-\mathrm{MoC}-(001)-\mathrm{Mo}$ & $-1.14\left({ }^{*} \mathrm{O}\right)$ & $-0.51\left({ }^{*} \mathrm{O}\right)$ & & \\
\hline$\eta-\mathrm{MoC}-(110)$ & $-0.37\left({ }^{*} \mathrm{OH}\right)$ & $-0.36\left({ }^{*} \mathrm{OH}\right)$ & $-0.60\left({ }^{*} \mathrm{OH}\right)$ & $-0.68\left({ }^{*} \mathrm{OH}\right)$ \\
\hline TiP-MoC-$-(110)$ & --- & $-0.37\left({ }^{*} \mathrm{OH}\right)$ & --- & $-0.35\left({ }^{*} \mathrm{OH}\right)$ \\
\hline TiP-MoC-$-(014)$ & --- & $-1.06\left({ }^{*} \mathrm{OH}\right)$ & --- & $-0.37\left({ }^{*} \mathrm{OH}\right)$ \\
\hline
\end{tabular}

Table S17 Zero-point energy (ZPE) corrections and entropy corrections to the free energies. We use gas phase $\mathrm{H}_{2} \mathrm{O}$ at 0.035 bars as reference state, as the gas phase $\mathrm{H}_{2} \mathrm{O}$ is in equilibrium with liquid water at $298.15 \mathrm{~K}$. The values for $\mathrm{H}_{2} \mathrm{O}$ and $\mathrm{H}_{2}$ are taken from CSRC book (Lide, D.R. CRC Hand book of Chemistry and Physics, CRC Press in 2003). The values of $\mathrm{E}_{\mathrm{ZPE}}$ for $* \mathrm{O}$ and $* \mathrm{OH}$ are calculated while they adsorb on $\delta-\mathrm{MoC}(001)$. The value of $\mathrm{E}_{\mathrm{ZPE}}$ for ${ }^{*} \mathrm{H}$ is different on $\mathrm{C}$ and $\mathrm{Mo}$ site, which is calculated on $\delta-\operatorname{MoC}(110)$. The corresponding values are also applied to other surfaces. The entropy corrections for ${ }^{*} \mathrm{H}, * \mathrm{O}$ and $* \mathrm{OH}$ are not considered.

\begin{tabular}{|c|c|c|c|c|c|c|}
\hline & $\mathrm{H}_{2}$ & $\mathrm{H}_{2} \mathrm{O}$ & ${ }^{*} \mathrm{H}(\mathrm{C})$ & ${ }^{*} \mathrm{H}(\mathrm{Mo})$ & ${ }^{*} \mathrm{OH}$ & ${ }^{*} \mathrm{O}$ \\
\hline $\mathrm{ZPE}$ & 0.27 & 0.56 & 0.24 & 0.17 & 0.35 & 0.08 \\
\hline $\mathrm{TS}$ & 0.41 & 0.67 & --- & --- & --- & --- \\
\hline
\end{tabular}

Table S18. In order to compare the $\mathrm{H}$ coverage on different surfaces, we also give the transformation of $\mathrm{H}$ coverage between unit of ML in the main text and unit of area $\left(\mathrm{N} / \mathrm{nm}^{2}\right)$ here for each surface. The corresponding number of " $\mathrm{N} / \mathrm{nm}^{2}$ " in this table indicates the $\mathrm{H}$ coverage at $1 \mathrm{H} \mathrm{ML}$ in the main text, e.g., the $1 \mathrm{H}$ ML is $41.94 \mathrm{H}$ atoms $/ \mathrm{nm}^{2}$ for $\delta-\mathrm{MoC}-001$.

\begin{tabular}{|c|c|c|c|c|c|c|c|c|c|c|}
\hline \multirow{2}{*}{$\begin{array}{l}\text { Phase } \\
\text { Surface }\end{array}$} & \multicolumn{4}{|c|}{$\delta-\mathrm{MoC}$} & \multicolumn{3}{|c|}{$\alpha-\mathrm{MoC}$} & \multicolumn{3}{|c|}{ G-MoC } \\
\hline & 001 & 110 & 111-Mo & $111-\mathrm{C}$ & $001-\mathrm{Mo} / \mathrm{C}$ & $100-\mathrm{C}$ & 110 & $\begin{array}{c}001- \\
\mathrm{Mo} / \mathrm{C}\end{array}$ & 110 & 010 \\
\hline$N / \mathrm{nm}^{2}$ & 41.94 & 14.83 & 12.11 & 12.11 & 54.42 & 12.14 & 28.04 & 13.20 & 14.49 & 25.10 \\
\hline Phase & \multicolumn{4}{|c|}{$\eta-\mathrm{MoC}$} & \multicolumn{3}{|c|}{ TiP-MoC } & & & \\
\hline Surface & 001-Мo & $001-\mathrm{C}$ & 110 & $0-14$ & 010 & 110 & 014 & & & \\
\hline$N / \mathrm{nm}^{2}$ & 12.37 & 12.37 & 14.71 & 14.01 & 24.68 & 14.25 & 20.00 & & & \\
\hline Phase & \multicolumn{6}{|c|}{$\beta-\mathrm{Mo}_{2} \mathrm{C}$} & \multicolumn{4}{|c|}{$\alpha-\mathrm{Mo}_{2} \mathrm{C}$} \\
\hline Surface & 100-Mo & $100-\mathrm{C}$ & 001-C & $110-\mathrm{C}$ & 110-Mo & 111-C & 001-C & 001-Mo & $100-\mathrm{C}$ & 011-C \\
\hline$N / \mathrm{nm}^{2}$ & 12.63 & 6.32 & 6.98 & 14.93 & 14.93 & 8.10 & 6.27 & 12.56 & 7.01 & 4.69 \\
\hline
\end{tabular}


a)

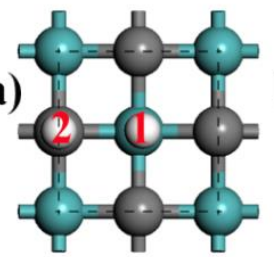

b)

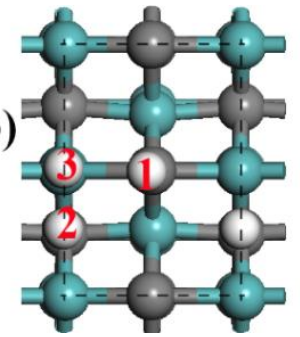

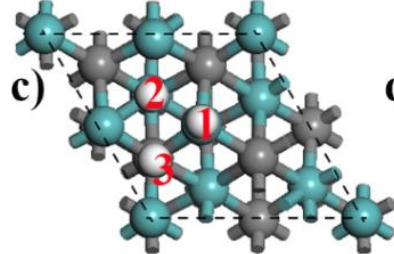

Lis tost

d)

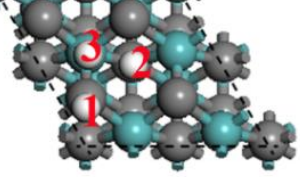

Figure S1 Possible adsorption sites for adsorbate on four different surfaces of $\delta-M o C$ : (a) (001), (b) (110), (c) (111)-Mo and (d) (111)-C. The adsorption site "1", "2" and "3" correspond to that in Table S2, and that of other $\mathrm{Mo}_{x} \mathrm{C}$ are in the same case. The blue, gray and white balls indicate Mo atom, $\mathrm{C}$ atom, and the possible adsorption site of adsorbate, respectively. The dashed line indicates the unit cell of slab in DFT calculations. The adsorbate indicates $\mathrm{H}, \mathrm{O}$, or $\mathrm{H}$. The corresponding number of atomic layers for (a), (b), (c), and (d) are 5, 5, 6, and 6, respectively.
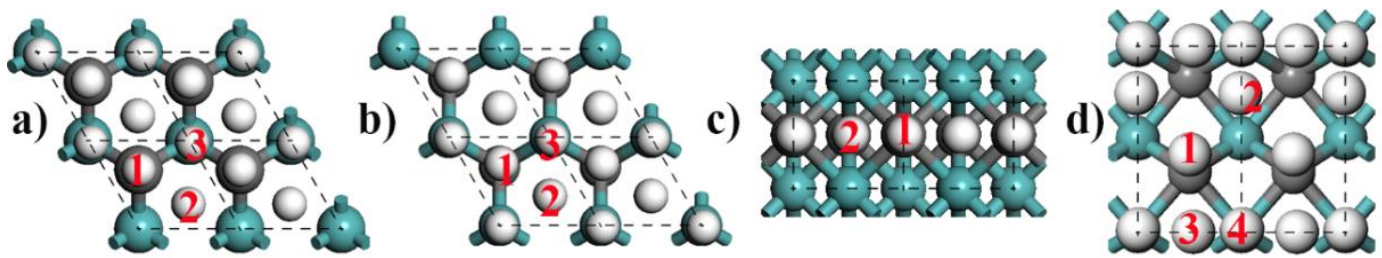

Figure S2 Possible adsorption sites for adsorbate on four different surfaces of $\alpha-\mathrm{MoC}$ : (a) (001)C, (b) (001)-Mo, (c) (100)-C and (d) (110). The corresponding number of atomic layers for (a), (b), (c), and (d) are 6, 6, 6, and 6, respectively.

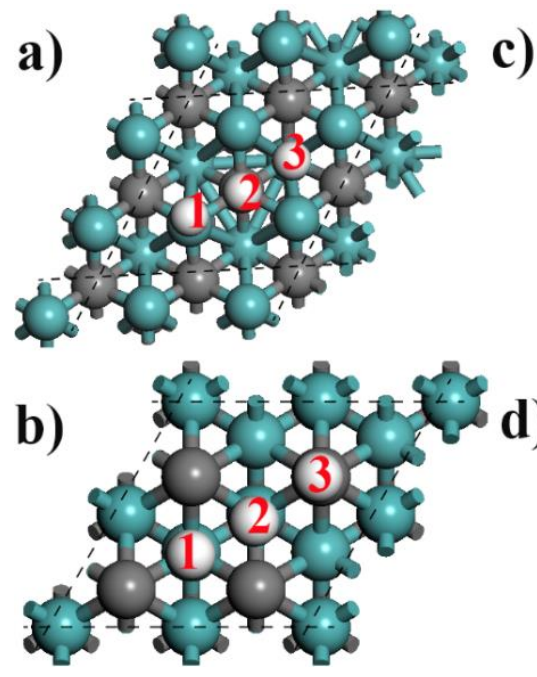

c)

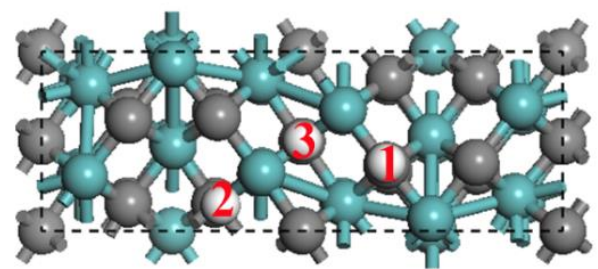

Figure S3 Possible adsorption sites for adsorbate on four surfaces of $\eta-\mathrm{MoC}$ : (a) (001)-Mo, (b) (001)-C, (c) (110) and (d) (0-14)-C. The corresponding number of atomic layers for (a), (b), (c), and (d) are 6, 6, 5, and 4, respectively. 
a)

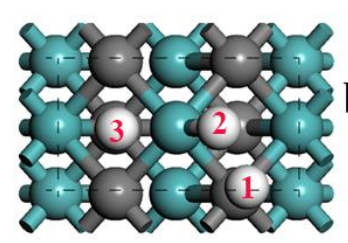

b)

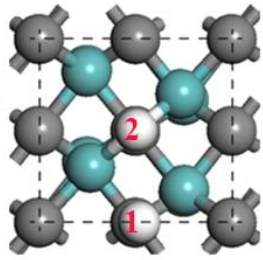

c)

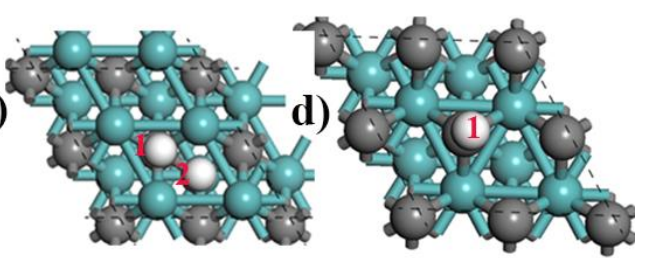

Figure S4 Possible adsorption sites for H atom on four surfaces of G-MoC: (a) (010), (b)110, (c) (001)-C and (d) (001)-Mo. The corresponding number of atomic layers for (a), (b), (c), and (d) are $5,9,6$, and 6 , respectively.

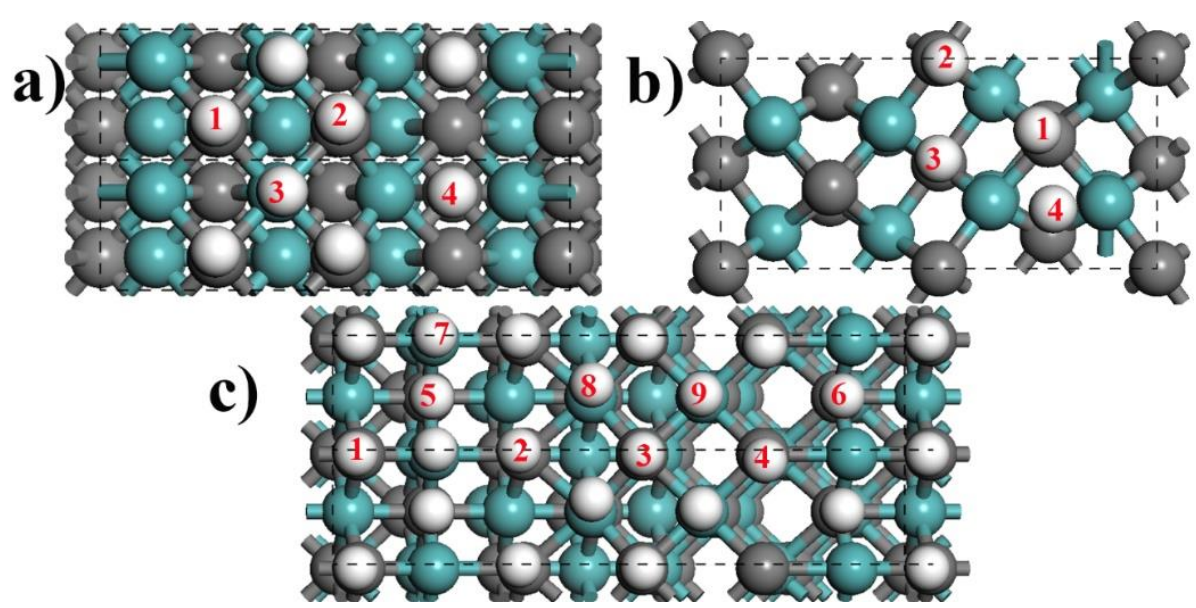

Figure S5 Possible adsorption sites for adsorbate on four surfaces of TiP-MoC: (a) (010) -Mo, (b) (110), (c) (014). The corresponding number of atomic layers for (a), (b), (c), and (d) are 4, 4, and 4, respectively.

a)
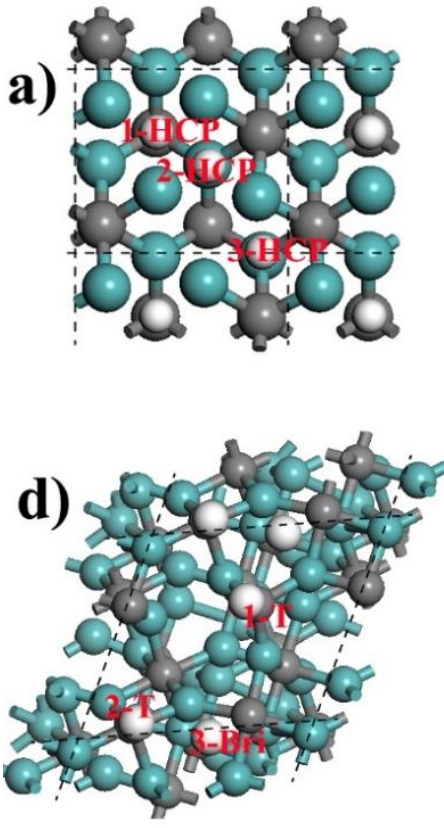

b)

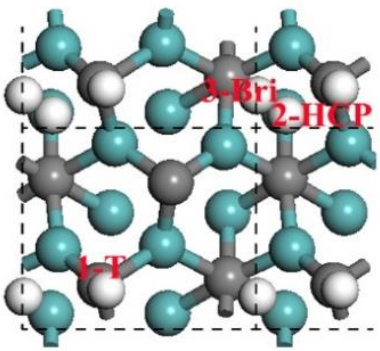

e)

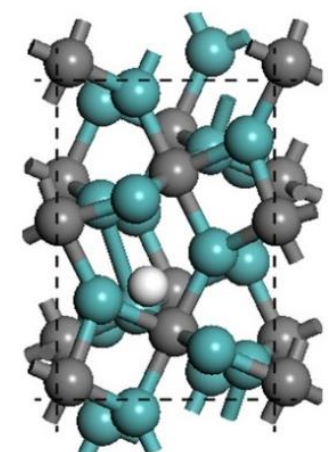

c)

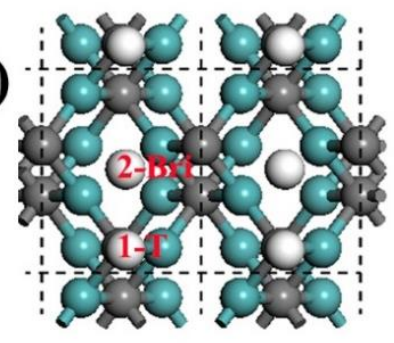

f)

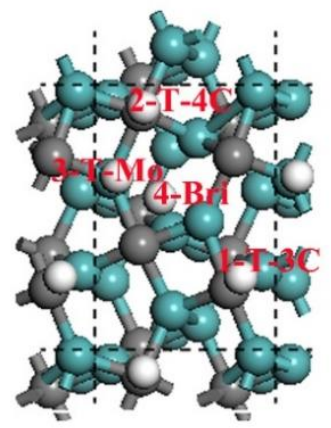

Figure S6 Possible adsorption sites for adsorbate on six different surfaces of $\beta-\mathrm{Mo}_{2} \mathrm{C}$ : (a) (100)Mo, (b) (100)-C, (c) (001)-C, (d) (111)-C, (e) (110)-Mo and (f) (110)-C. The corresponding number of atomic layers for (a), (b), (c), (d), (e) and (f) are 4, 4, 9, 8, 8, and 9, respectively. 


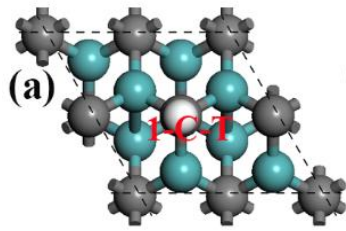

(b)

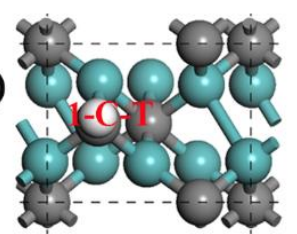

(c)

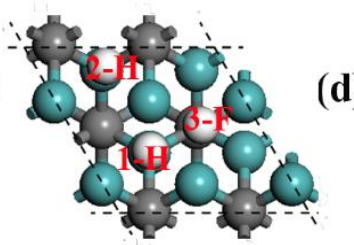

(d)

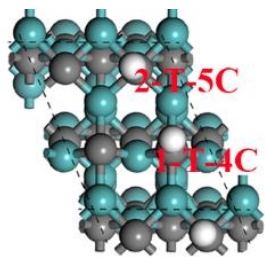

Figure S7 Possible adsorption sites for adsorbate on our different surfaces of $\alpha-\mathrm{Mo}_{2} \mathrm{C}$ : (a) (001)C, (b) (100)-C, (c) (001)-Mo and (d) (011). The corresponding number of atomic layers for (a), (b), (c), and (d) are $6,6,6$, and 9 , respectively.

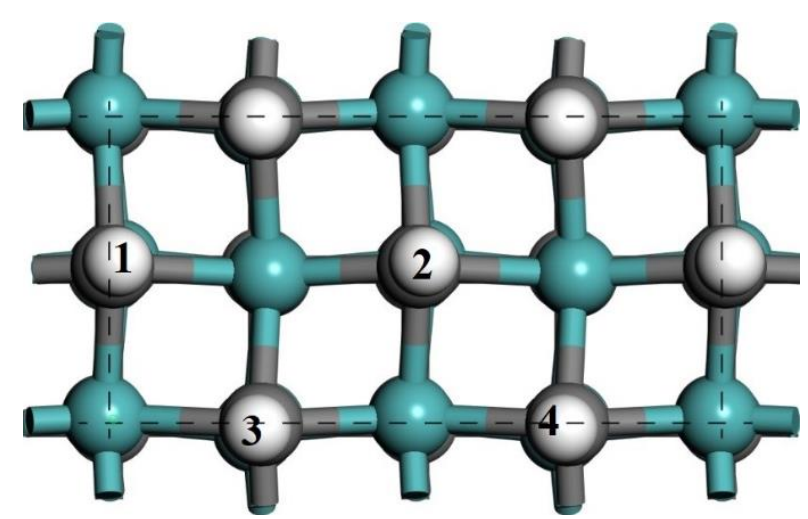

Figure S8 The adsorption process of $\mathrm{H}$ atoms on $\delta-\operatorname{MoC}(001)$. The "1", "2", "3" and " 4 " indicate the first, second, third, and fourth $\mathrm{H}$ atom in adsorption process, or the $\mathrm{H}$ coverage of $\delta-\mathrm{MoC}(001)$ $1 / 8,2 / 8,3 / 8$, and $4 / 8 \mathrm{H}$ ML as shown in Figure 3a in main text.

a)

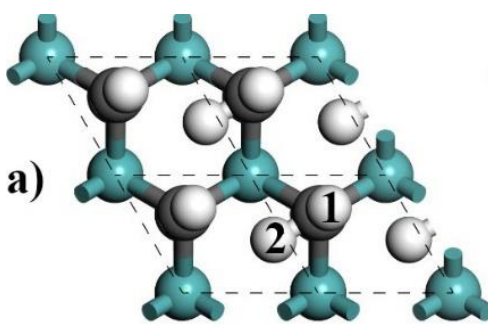

b)

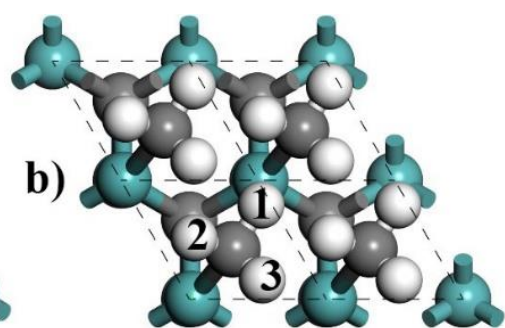

Figure S9 The adsorption process of $\mathrm{H}$ atoms on $\alpha-\operatorname{MoC}(001)-\mathrm{C}$. The (a) and (b) represent the corresponding surface adsorbs two and three $\mathrm{H}$ atoms. The "1", "2", and " 3 " and indicate the first, second, and third $\mathrm{H}$ atom in adsorption process, or the $\mathrm{H}$ coverage of $\alpha-\mathrm{MoC}(001)-\mathrm{C} 1,2$, and $3 \mathrm{H}$ ML as shown in Figure 3b in main text. It should be noted that, there is larger structural deformation from the (a) to (b)." 


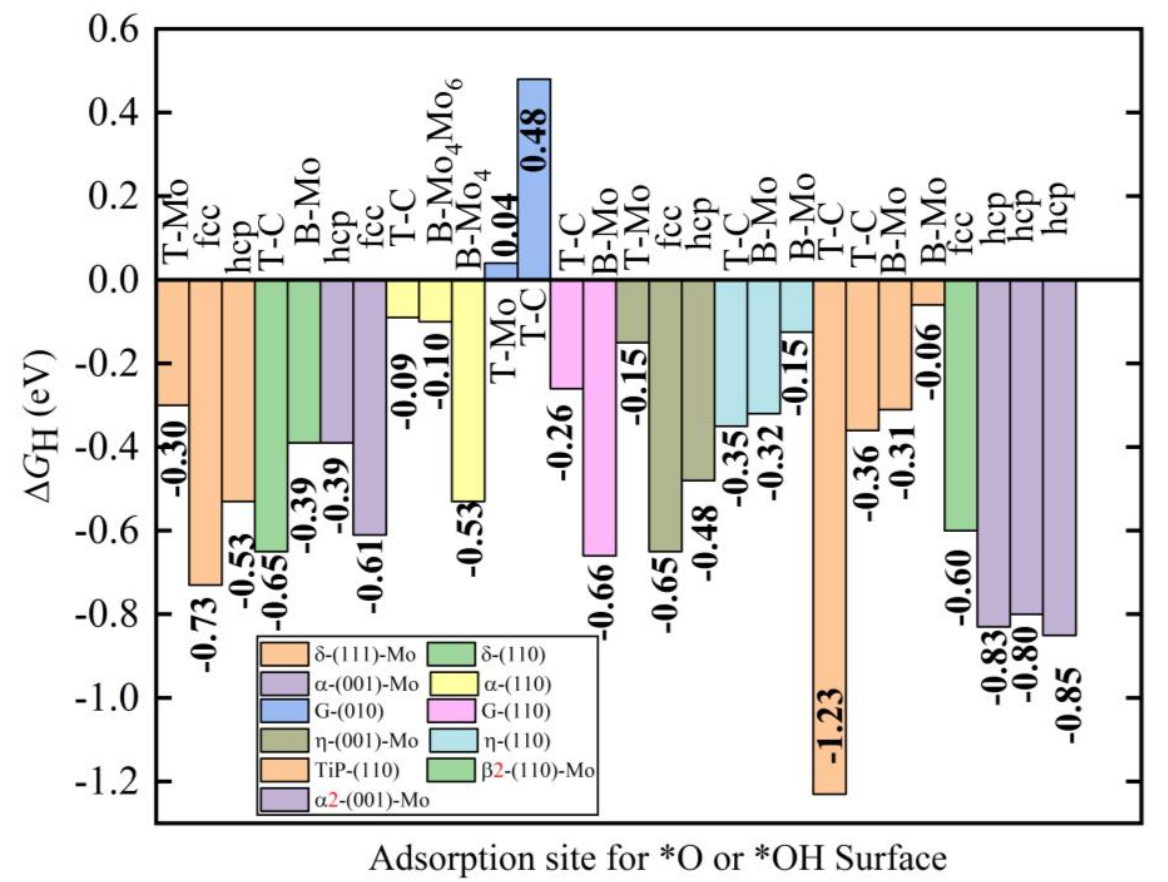

Figure S10 The free energy change of adsorbed $\mathrm{H}\left(\Delta \mathrm{G}_{\mathrm{H}}\right)$ on different sites of bare surfaces. Those surfaces are corresponding surface in Figure 4a while not considering water effect. And the corresponding $\Delta \mathrm{G}_{\mathrm{H}}$ are from Table S2-S8.

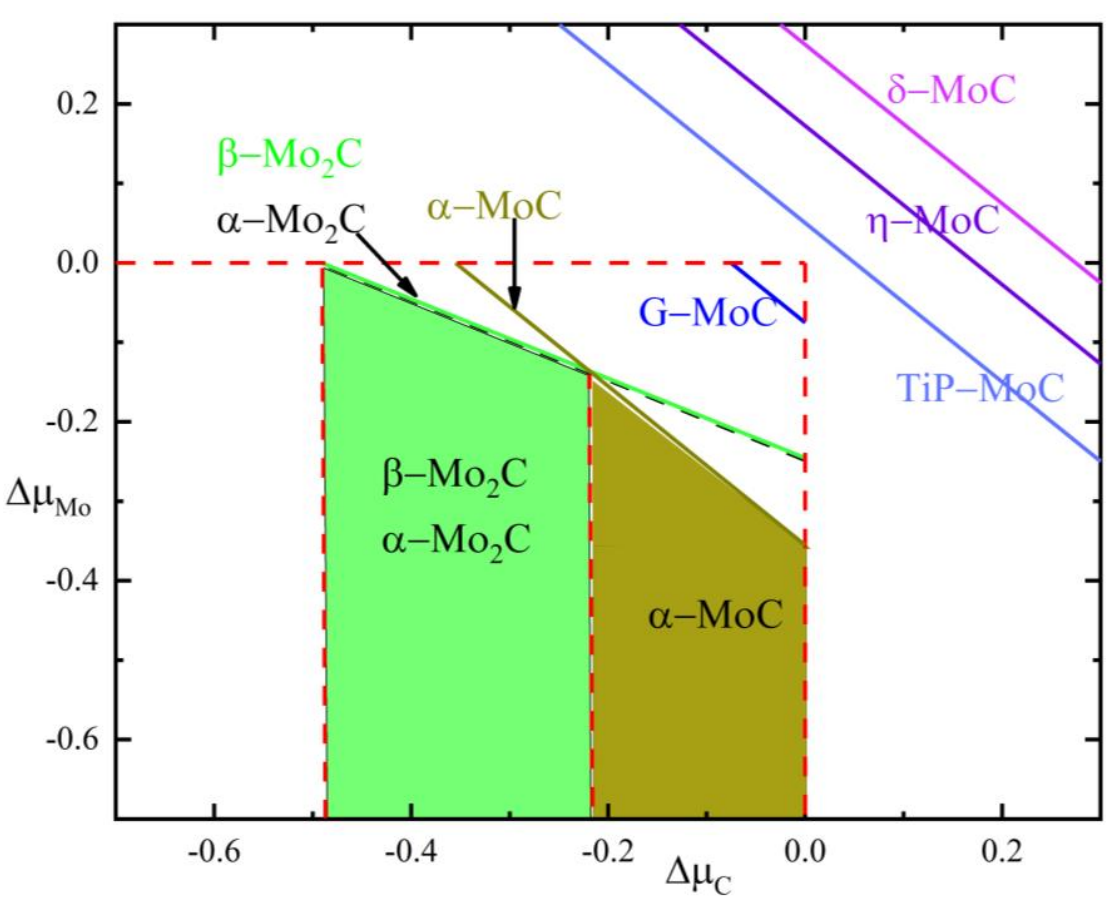

Figure S11 Bulk phase diagram as function of the relative potential of $\mathrm{C}$ and Mo for different phases of molybdenum carbides. The relative chemical potential $\left(\Delta \mu_{\mathrm{C}}\right.$ and $\triangle \mu_{\mathrm{mo}}$ ) is defined as that relative to the chemical potential in element bulk ( $\mathrm{C}$ in diamond bulk and fcc Mo bulk, respectively). It clearly shows that the $\alpha-\mathrm{MoC}$ will be stable at - 
$0.22 \mathrm{eV} \leq \Delta \mu_{\mathrm{C}} \leq 0 \mathrm{eV}$. The two phases of $\mathrm{Mo}_{2} \mathrm{C}$ will be stable at $-0.49 \mathrm{eV} \leq \triangle \mu_{\mathrm{C}} \leq-0.22 \mathrm{eV}$. The $\delta-\mathrm{MoC}, \eta-\mathrm{MoC}$ and TiP-MoC are not stable in this calculation as the entropy contributions are not taken into considerations as $\mathrm{T}=0 \mathrm{~K}$ in this calculation.
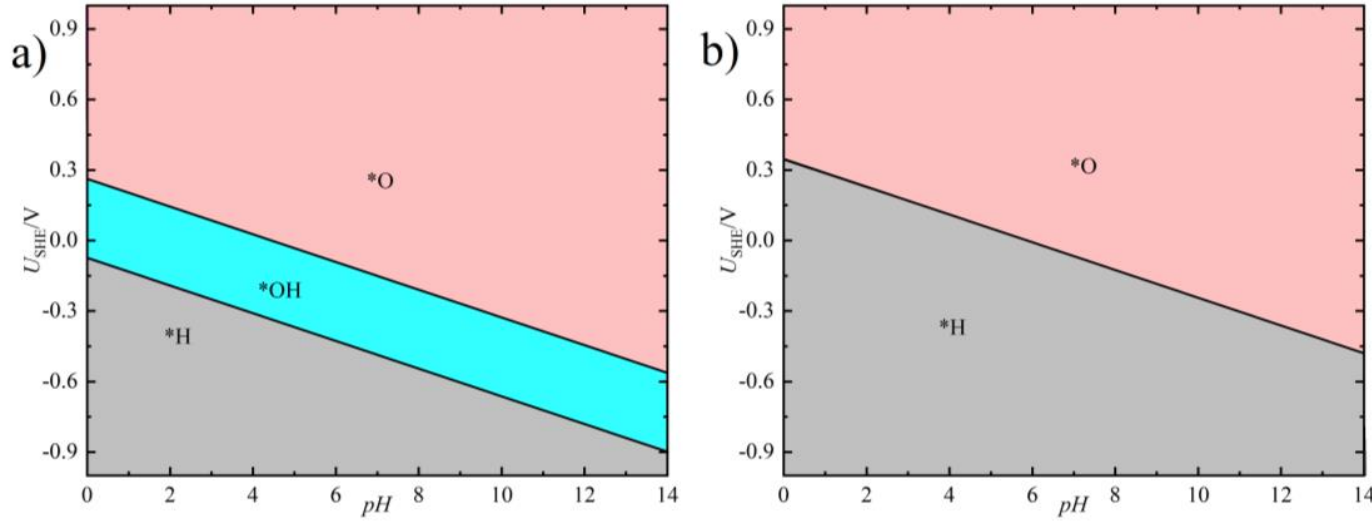

Figure S12 Computed surface phase diagram for (a) (110) and (b) (100)-C of $\alpha-\mathrm{MoC}$ in aqueous solution. The colored areas indicate corresponding surface phase. The "*H", “* $\mathrm{OH}$ " and "*O" represent corresponding surface tends to covered by adsorbed $\mathrm{H}, \mathrm{OH}$ and $\mathrm{O}$ at given condition $\left(\mathrm{pH}\right.$ and electrode potential $\mathrm{U}_{\mathrm{SHE}}$ ), respectively. The corresponding data are from Table S3 and S10. The corresponding surfaces calculated are as the slab model in Figure S2c and S2d with one adsorbing one $\mathrm{H}, \mathrm{OH}$ or $\mathrm{O}$, respectively. The corresponding method applied are from ref.58 in main text. It could be seen from (a) that the adsorbed $\mathrm{OH}$ could form in a relative smaller region in (110), e.g., the $\mathrm{U}_{\mathrm{SHE}}$ will be $-0.07 \mathrm{eV}<\mathrm{U}_{\mathrm{SHE}}<0.26$ while $\mathrm{pH}=0$. In the $(100)-\mathrm{C}$, the ${ }^{*} \mathrm{OH}$ will be not formed in any case, and the ${ }^{*} \mathrm{O}$ will occur in alkaline solution.

\section{REFERENCE}

1. Klimeš, J.; Bowler, D. R.; Michaelides, A., Van Der Waals Density Functionals Applied to Solids. Physical Review B 2011, 83, 195131.

2. Guillermet, A. F.; Häglund, J.; Grimvall, G., Cohesive Properties of 4d-Transition-Metal Carbides and Nitrides in the NaCl-Type Structure. Physical Review B 1992, 45, 11557.

3. Viñes, F.; Sousa, C.; Liu, P.; Rodriguez, J.; Illas, F., A Systematic Density Functional Theory Study of the Electronic Structure of Bulk and (001) Surface of Transition-Metals Carbides. The Journal of Chemical Physics 2005, 122, 174709.

4. Chrysanthou, A.; Grieveson, P., The Observation of Metastable Molybdenum Carbides. Journal of Materials Science Letters 1991, 10, 145-146.

5. Zheng, W.; Cotter, T. P.; Kaghazchi, P.; Jacob, T.; Frank, B.; Schlichte, K.; Zhang, W.; Su, D. S.; Schuith, F.; Schlogl, R., Experimental and Theoretical Investigation of Molybdenum Carbide and 
Nitride as Catalysts for Ammonia Decomposition. Journal of the American Chemical Society 2013, 135, 3458-3464.

6. Clougherty, E.; Lothrop, K.; Kafalas, J., A New Phase Formed by High-Pressure Treatment: Face-Centred Cubic Molybdenum Monocarbide. Nature 1961, 191, 1194.

7. Otani, S.; Ishizawa, Y., Preparation of $\mathrm{Mo}_{2} \mathrm{C}$ Single Crystals by the Floating Zone Method. Journal of crystal growth 1995, 154, 202-204.

8. Haines, J.; Leger, J.; Chateau, C.; Lowther, J., Experimental and Theoretical Investigation of $\mathrm{Mo}_{2} \mathrm{C}$ at High Pressure. Journal of Physics: Condensed Matter 2001, 13, 2447. 